\section{Basket cases}

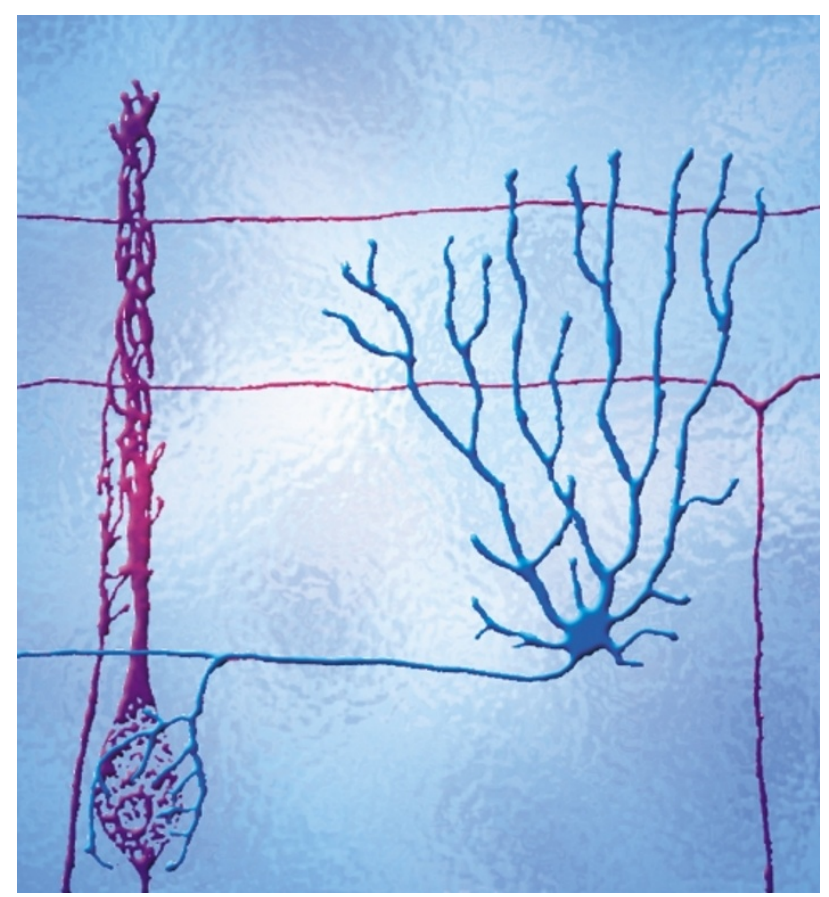

Basket cells — inhibitory interneurons that are found in the neocortex, cerebellum and hippocampus, and form 'baskets' of axonal arborizations onto and around the somata of target neurons - were first described in 1911 by Ramon y Cajal. Since then, it has become clear that there are different types of basket cell with diverse structural and functional properties, but researchers have lacked a detailed classification of these cells. Wang and colleagues have investigated the properties of these neurons in the cortex in unprecedented detail, and conclude that there are three distinct classes of cortical basket cell.

It has generally been accepted that there are two 'classical' subtypes of basket cell — small and large (SBCs and LBCs) — which are distinguished by the morphologies of their axonal arborizations. Many studies have described other basket cells that did not fit into either of these classifications and, in 2000, Gupta et al. named these 'nest' basket cells (NBCs). Until now, the number and importance of these cells in the cortex has been unclear, but new data published in Cerebral Cortex show that NBCs are the most common type of basket cell in layers II/III of the somatosensory cortex, and that they are a powerful inhibitory force in these layers.

Wang et al. used a number of different techniques to characterize basket cells, including electrophysiology, microscopy and single-cell polymerase chain reaction (PCR) analysis of messenger RNA expression. They found that NBCs differed from the other two classes of basket cell not only in their morphology, but also in their electrophysiological responses, which were diverse, and in their molecular characteristics. Each class of basket cell showed a different pattern of mRNA expression; for example, NBCs and LBCs could be distinguished from SBCs because SBCs invariably expressed the mRNA for vasoactive intestinal peptide (VIP), whereas NBCs and LBCs never did.

Overall, $50 \%$ of the basket cells in layers II/III were NBCs. Although their electrophysiological properties were heterogeneous, a common

SYNAPTIC PLASTICITY

\title{
Remember PKC?
}

You are packed in a dimly lit, narrow hallway. It is hot and the air feels heavy from the humidity. The current of air that runs through suddenly changes to a sharp stench that can only be compared to sweaty socks. Then it happens - a current of electricity runs across the floor and jolts you over and over. A second, equally foul odour follows. You prepare for the worst, but nothing happens. Suddenly, you are slammed sideways and crammed into an even smaller space. After some jostling, the space opens into another hall. From one direction you smell socks; from the other, that second foul odour. Where to go? This is what a fly might experience in an odouravoidance learning task that was used in one of two papers in Nature Neuroscience to unravel the role of protein kinase $\mathrm{C}$ (PKC) in memory and long-term potentiation (LTP).

There are three main categories of PKC molecules - conventional, novel and atypical. To add another layer of complexity, these kinases can exist in two forms - a full-length form and a truncated form that lacks the amino-terminal regulatory domain, leaving a constitutively active catalytic domain (PKM). There is evidence that one form in particular - atypical PKM $\zeta$ - is maintained at an elevated level during the maintenance phase of LTP. But what is it doing? Drier et al. obtained evidence that the acute expression of mouse or Drosophila PKM $\zeta$ in flies enhances memory formation after olfactory avoidance training. Curiously, PKM $\zeta$ was not able to enhance long-term memory formation, but only affected a proteinsynthesis-independent form that is referred to as anaesthesia-resistant memory. The authors argue that the enhancement of longterm memory might not be detected if $\mathrm{PKM} \zeta$ is crucial for memory maintenance, because the endogenous enzyme would already have been activated by training.

The experiments by Drier et $a l$. are in agreement with work done at the cellular level by Ling et al., who show that PKM $\zeta$ can maintain LTP over a six-hour period in rat hippocampal slices. Injection of mouse PKM $\zeta$ into postsynaptic neurons elicited a potentiation of transmission that occluded LTP induced by synaptic activity. Moreover, manipulation of enzymatic function using a dominant-negative form of PKM $\zeta$ enabled Ling et al. to block both the PKM $\zeta$-induced potentiation and the activity-dependent LTP.

The results of these two studies indicate that PKM $\zeta$ has an essential role in LTP and memory maintenance, adding another degree of freedom to the molecular mechanisms that underlie synaptic plasticity, a field in which the focus has been centred largely on other protein kinases, such as calcium/calmodulin-dependent protein kinase II (CaMKII). Michael Stebbins
Assistant Editor, Nature Genetics (2) References and links ORIGINAL RESEARCH PAPERS Ling, D. S. F. et al. Protein kinase $M \zeta$ is necessary and sufficient for LTP maintenance. Nature Neurosci. 5, 295-296 (2002) | Drier, E. A. et al. Memory enhancement and formation by atypical PKM Memory enhancement and formation by atypical PKM
activity in Drosophila melanogaster. Nature Neurosci. $\mathbf{5}$ activity in Drosop FURTHER READING Paulsen, O. \& Morris, R. Flies put the buzz back into long-term-potentiation. Nature Neurosci. $\mathbf{5}$ 289-290 (2002) 
feature was that they seemed to be 'high-threshold' interneurons many pyramidal neurons are needed to recruit each NBC. The authors conclude, however, that once recruited, NBCs provide most of the perisomatic inhibition in these layers.

It is clear that such a precise and thorough classification of neurons is vital if we are to approach a full understanding of neural circuitry and interactions in any area of the brain. This kind of multidisciplinary approach will be important in future studies of neuronal classification. In particular, it will be interesting to see whether the properties of basket cells in the cerebellum and hippocampus reflect those found in the cortex.

Rachel Jones

(2) References and links ORIGINAL RESEARCH PAPER Wang, Y. et al. Anatomical, physiological, molecular and circuit properties of nest basket cells in the developing somatosensory cortex. Cereb. Cortex 12, 395-410 (2002)

FURTHER READING McBain, C. J. \& Fisahn, A. Interneurons unbound. Nature Rev. Neurosci. 2 , 11-23 (2001) WEB SITES

Markram's lab: http://www.weizmann.ac.il/ neurobiology/labs/markram/markram.html

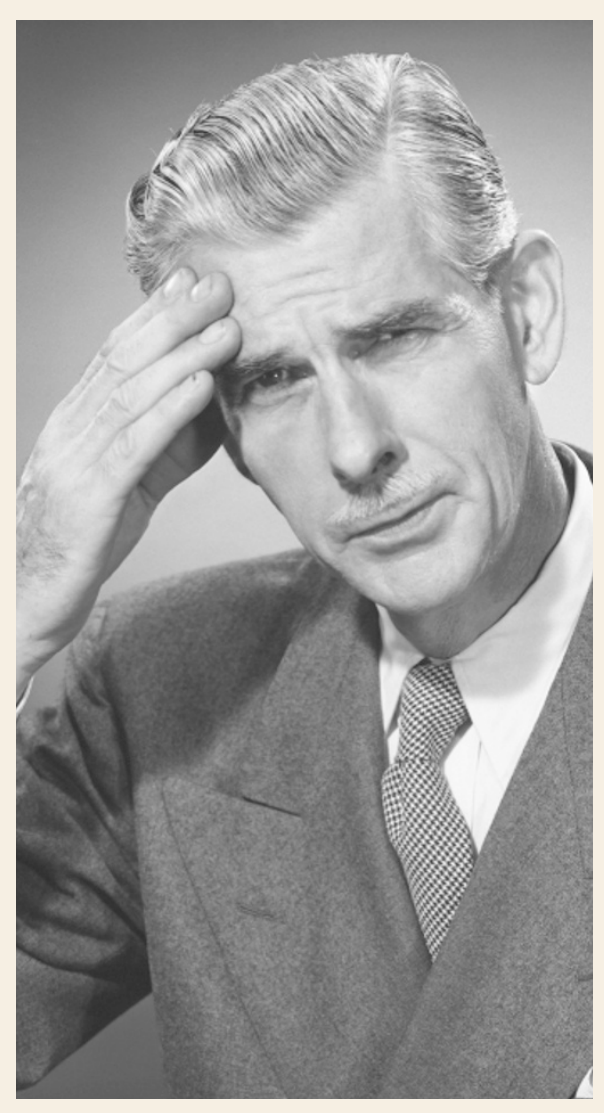

\section{BEHAVIOURAL GENETICS}

\section{High anxiety}

The function of serotonin as a mood regulator is well known, and both serotonin itself and drugs that activate its signalling pathway have been used successfully to treat depression and anxiety disorders. However, the role of serotonin in establishing normal responses to anxiety-inducing stimuli has been unclear. Now, by manipulating the expression of a serotonin receptor in mice, Gross et al. have brought us one step closer to understanding this role.

To measure anxiety levels in mice, the authors studied their behaviour in an open field or an 'elevated-plus maze'. Mice generally prefer to be in a 'safe' environment, such as in the closed arms of the maze or at the sides of the open-field box, but their natural curiosity causes them to explore more aversive environments, such as the open arms of the maze or the centre of the open field. Their level of anxiety is determined by the amount of time that they spend exploring the aversive compartment of the apparatus; the more adventurous the mouse, the less anxious it is perceived to be.

It was previously shown that a global knockout of the serotonin ${ }_{1 \mathrm{~A}}$ receptor (5-HT1AR) causes an increase in anxiety-like behaviour in adult mice. By engineering a conditional knockout line in which the receptor gene could be selectively reactivated in the forebrain, Gross et al. were able to rescue this phenotype, allowing the mice to behave normally in response to anxiety-inducing situations. This result indicated that 5-HT1AR activity at its other main site of expression, the raphe nuclei of the brainstem, is not needed to reverse the anxiety phenotype in the knockout mice.

Another property of this 'rescued' mouse line was that 5-HT1AR expression could be switched off again by treating the mice with the antibiotic doxycycline. Gross et al. compared the effects of inactivating the receptor during late embryonic/early postnatal development and during adulthood. If the receptor was inactivated in adult mice, their responses to anxiety-inducing stimuli were indistinguishable from those of wild-type mice. By contrast, if the receptor was inactivated during development, the adult mice behaved like knockout mice, even when 5-HT1AR expression was restored at postnatal day 21 . This result, combined with the observation that 5-HT1AR expression begins at postnatal day 5 in the rescued mice, indicates that there is a critical period between 5 and 21 days after birth when 5-HT1AR is required in the forebrain to establish normal anxiety responses. Failure to express the receptor during this period causes the mice to become excessively anxious in later life, but sustained expression in the adult mouse is not necessary to maintain a normal anxiety response.

The mechanism underlying this effect is as yet unknown, although 5-HT1AR has previously been implicated in synaptogenesis in the hippocampus and cortex, so it could be involved in establishing the neuronal circuits that are required for normal anxiety responses. Interestingly, in adults, chronic treatment with 5-HT1AR agonists can decrease anxiety, raising the possibility that similar mechanisms can be triggered in adulthood. Understanding these mechanisms should help in the design of more sophisticated therapies to treat anxiety disorders. It will also be interesting to look at factors that are believed to influence emotional well-being, such as maternal nurturing, and examine whether they affect 5-HT1AR activity during early life.

Heather Wood

(4) References and links

ORIGINAL RESEARCH PAPER Gross, C. et al. Serotonin receptor acts during development to establish normal anxiety-like behavior in the adult. Nature 416, 396-400 (2002)

FURTHER READING Snyder, S. H. Serotonin sustains serenity. Nature 416, 377-380 (2002)

\section{WEB SITES}

Encyclopedia of Life Sciences: http://www.els.net

serotonin receptors 